\title{
Multifactorial analysis of the stochastic epigenetic variability in cord blood confirmed an impact of common behavioral and environmental factors but not of in vitro conception
}

D. Gentilini ${ }^{1,5}$, E. Somigliana ${ }^{2}$, L. Pagliardini ${ }^{3}$, E. Rabellotti ${ }^{3}$, P. Garagnani ${ }^{4}$, L. Bernardinelli ${ }^{5}$, E. Papaleo $^{3}$, M. Candiani ${ }^{6}$, A. M. Di Blasio ${ }^{1}$ and P. Viganò ${ }^{3^{*}}$

\begin{abstract}
Background: An increased incidence of imprint-associated disorders has been reported in babies born from assisted reproductive technology (ART). However, previous studies supporting an association between ART and an altered DNA methylation status of the conceived babies have been often conducted on a limited number of methylation sites and without correction for critical potential confounders. Moreover, all the previous studies focused on the identification of methylation changes shared among subjects while an evaluation of stochastic differences has never been conducted. This study aims to evaluate the effect of ART and other common behavioral or environmental factors associated with pregnancy on stochastic epigenetic variability using a multivariate approach.

Results: DNA methylation levels of cord blood from 23 in vitro and 41 naturally conceived children were analyzed using the Infinium HumanMethylation450 BeadChips. After multiple testing correction, no statistically significant difference emerged in the number of cord blood stochastic epigenetic variations or in the methylation levels between in vitro- and in vivo-conceived babies. Conversely, four multiple factor analysis dimensions summarizing common phenotypic, behavioral, or environmental factors (cord blood cell composition, pre or post conception supplementation of folates, birth percentiles, gestational age, cesarean section, pre-gestational mother's weight, parents' $\mathrm{BMI}$ and obesity status, presence of adverse pregnancy outcomes, mother's smoking status, and season of birth) were significantly associated with stochastic epigenetic variability. The stochastic epigenetic variation analysis allowed the identification of a rare imprinting defect in the locus GNAS in one of the babies belonging to the control population, which would not have emerged using a classical case-control association analysis.

Conclusions: We confirmed the effect of several common behavioral or environmental factors on the epigenome of newborns and described for the first time an epigenetic effect related to season of birth. Children born after ART did not appear to have an increased risk of genome-wide changes in DNA methylation either at specific loci or randomly scattered throughout the genome. The inability to identify differences between cases and controls suggests that the number of stochastic epigenetic variations potentially induced by ART was not greater than that naturally produced in response to maternal behavior or other common environmental factors.
\end{abstract}

Keywords: Epigenome-wide analysis, Assisted reproduction technology, DNA methylation, Imprinted genes, Multiple factor analysis, Stochastic epigenetic variations

\footnotetext{
*Correspondence: vigano.paola@hsr.it

${ }^{3}$ Reproductive Sciences Laboratory, Division of Genetics and Cell Biology,

IRCCS Ospedale San Raffaele, Via Olgettina 58, 20132 Milan, Italy

Full list of author information is available at the end of the article
}

(c) The Author(s). 2018 Open Access This article is distributed under the terms of the Creative Commons Attribution 4.0 International License (http://creativecommons.org/licenses/by/4.0/), which permits unrestricted use, distribution, and reproduction in any medium, provided you give appropriate credit to the original author(s) and the source, provide a link to the Creative Commons license, and indicate if changes were made. The Creative Commons Public Domain Dedication waiver (http://creativecommons.org/publicdomain/zero/1.0/) applies to the data made available in this article, unless otherwise stated. 


\section{Background}

Currently, there is still poor consensus on the possibility that assisted reproduction technology (ART) could affect the epigenome of in vitro-conceived babies [1-3].

Most of the studies addressing the DNA methylation status of children conceived through in vitro fertilization (IVF) with or without intracytoplasmic sperm injection (ICSI) have specifically evaluated alterations in imprinted regions $[1,2]$. This on the basis of a suggested link between ART and imprinting disorders. A recent meta-analysis including the results of all the studies regardless of the type of the imprinting disorder, showed a significant association between imprinting diseases and ART (odds ratio $=3.67$; 95\% confidence interval $=1.39-9.74) \quad[2]$. Beckwith-Wiedemann syndrome, as an example, has an estimated worldwide frequency of 1 in 13,700 naturally conceived babies and a weighted relative risk of 5.2 in children conceived by ART $[2,4]$. Therefore, there is evidence to suggest an adverse effect of ART procedures, as well as of the underlying subfertility, on imprinting status. The biological rationale behind this idea is that because of the dynamic epigenetic reprogramming occurring during oocyte growth and preimplantation development, environmental perturbations during this time period may affect imprinting establishment and maintenance. Proper allelic expression of imprinted genes are known to play an important role in embryonic and neonatal growth, placental function, and postnatal behavior $[1,2,4-7]$.

On the other hand, no evidence of generalized changes in DNA methylation of the imprinted genes KvDMR/ KCNQIOTI, PEGI/MESR, IGF2, PEG3, and H19 in association with ART was found [2]. For instance, results of a meta-analysis of four studies comparing percentage methylation of IGF2 locus between ART and spontaneously conceived babies did not show any significant difference [2]. Although imprinting syndromes are usually associated with profound methylation changes, even the smaller scale changes that could be caused by ART methodologies could potentially predispose to epigenetic alterations at the key loci associated with the syndromes. However, it is unlikely that these hypothetical changes in the methylation status of newborns will be located in genomic regions shared among subjects. Considering that the reprogramming mechanism is driven by a relatively small number of "players" [8], it is difficult to figure out a mechanism where a potential interference related to IVF techniques will result in a small localized damage instead of a stochastic and more widespread effect. Indeed, the stochastic nature of these events has been previously suggested based on data both in humans and animal models $[9,10]$. In addition to a potential damage, a stochastic effect could also be related to a rescue mechanism similar to the one observed in the germinal cells of the progeny of Dnmt3L2/2 female mice, where a hierarchy of factors involved in the establishment and maintenance of maternal germline imprints has been found, the loss of one possibly rescued in a stochastic fashion by the activity of the others [11].

Given the supposed small and stochastic effects associated with ART, the analysis of mean methylation levels may not be ideal in exploring differences between in vivo- and in vitro-conceived children. This analysis may be useful in identifying epigenetic alterations shared by a group of subjects and potentially associated with their phenotype but would not reflect differences in individual variation or other features of the methylation spectrum. Rare stochastic epigenetic variations that are not shared among subjects fail to be identified by a classic Epigenome Wide Association Study (EWAS) based on mean methylation values comparison [12]. To overcome this problem, we have conducted an analysis of stochasticity based on the previously introduced concept of stochastic epigenetic variations (SEVs) [12]. SEV is defined as a single $\mathrm{CpG}$ with a methylation level detected as an outlier when compared to the methylation level found for the same CpG in a control population. This allows to obtain both a measure of stochasticity at a whole-genome level (total number of SEVs) and a topographic information on genomic regions with an enriched number of SEVs. A previous application of this method permitted to obtain an estimation of the number of epigenetic alterations produced by aging, revealing an exponential association between age and number of SEVs [12]. Only four studies have previously evaluated DNA methylation at a genome-wide level in cord blood of human offspring from ART procedures, and this was done based on the comparison of the mean methylation levels [10, 13-15]. Importantly, correction for phenotypic parental or fetal traits was limited to none or very few confounders although parental BMI, mode of delivery, birth weight, smoke, and intrauterine growth restriction have been previously linked to changes in methylation levels of several genes [16-19].

In this report, we present different analytical approaches in evaluating global DNA methylation of umbilical cord blood from in vitro and naturally conceived newborns mostly with the aim to measure random epigenetic variations and the impact of potential confounders on their number.

\section{Results}

\section{Dimensionality reduction of all phenotypic traits using} multiple factor analysis

Differences at phenotypic level between naturally conceived $(n=41)$ and in vitro-conceived babies $(n=23)$ were evaluated. The phenotypic traits considered were birth weight, birthweight centiles, mother's age, parity status, gestational age, cesarean section, sex of the baby, 
presence of adverse pregnancy outcomes (diabetes, preeclampsia and placenta praevia), pre-gestational mother weight, parents' BMI and obesity, gestational weight increase, maternal smoking status, pre-post conception folate supplementation, blood cell composition [CD8 T Cells (CD8T), CD4 T Cells (CD4T), natural killer (NK) cells, B cells, monocytes, granulocytes], and season of birth. The correlogram in Additional file 1: Figure S1 shows all the phenotypes analyzed and the presence of a consistent degree of correlation among traits. The number of dimensions to use in the analysis was reduced using the multiple factor analysis of mixed data. Considering the first two obtained dimensions (Dims), the overlap of cases and controls shown in Fig. 1a suggested a phenotypic similarity between the two groups. Taken together, the first 10 dimensions collected $72 \%$ of the total variability among the subjects analyzed. Correlations between dimensions and phenotypic traits were evaluated and are shown in Fig. 1b. The logistic regression analysis indicated that the Dim 1 and Dim 3 were significantly associated with case-control status $(p=0.03$ and $p=$ 0.01 , respectively) and were subsequently used as covariates in the case-control differential methylation analysis.
Phenotypic traits that were mainly captured by Dim1 and Dim 3 were highlighted by elevated correlation levels (Fig. 1b).

\section{Visual inspection of DNA methylation level using principal component analysis (PCA)}

The genome-wide DNA methylation analysis of cord blood was performed using the Infinium HumanMethylation450 BeadChip. Dimensional reduction was used to visually inspect the dataset for strong signals in the methylation values. The PCA was performed considering methylation signals from single $\mathrm{CpG}$ sites and also considering four sets of genomic regions: genes, promoters, $\mathrm{CpG}$ islands, and tiling (not overlapping regions of $5 \mathrm{~kb}$ length). Results reported in Fig. 2 show that there was no strong difference in the methylation level between naturally conceived and in vitro-conceived babies considering both sites and genomic regions.

\section{Differential methylation analysis}

Differential methylation analysis was computed both for site (single $\mathrm{CpG}$ ) and region level. Dimensions obtained from the multifactorial analysis, which were significantly

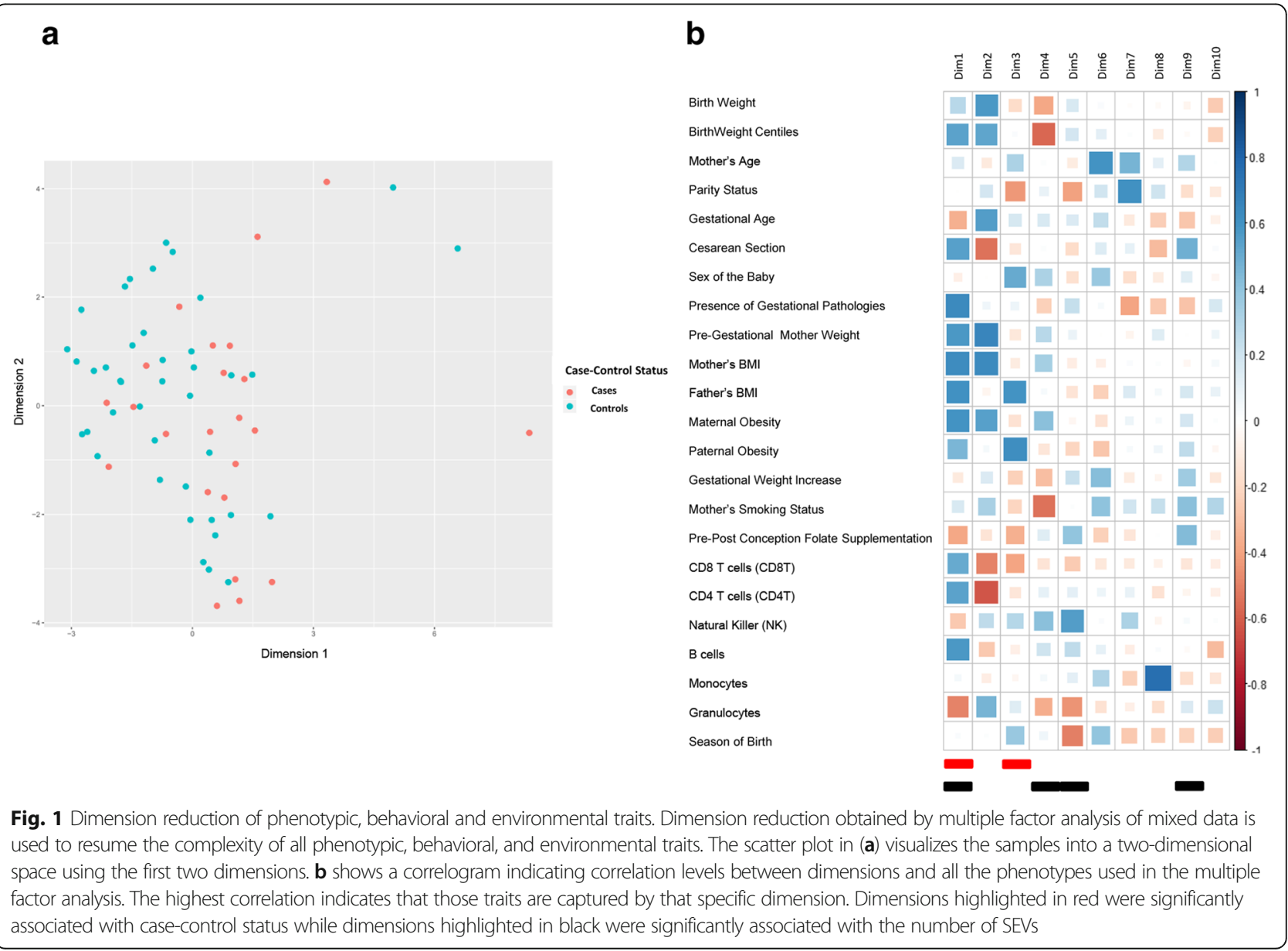




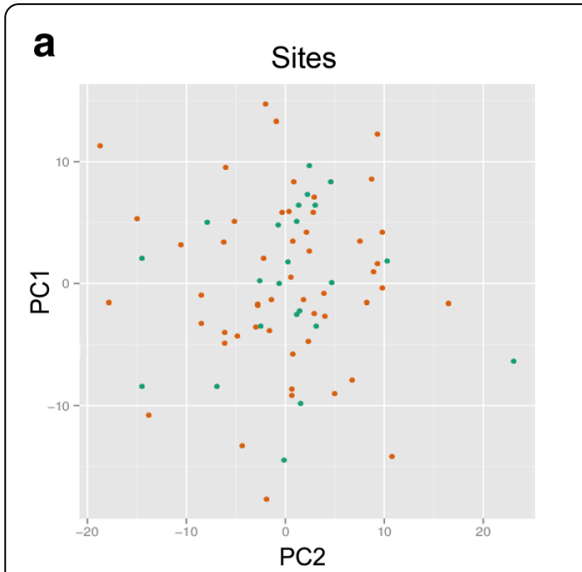

b

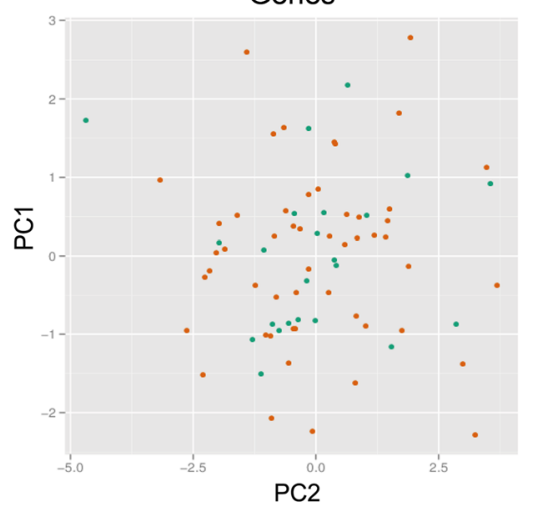

c

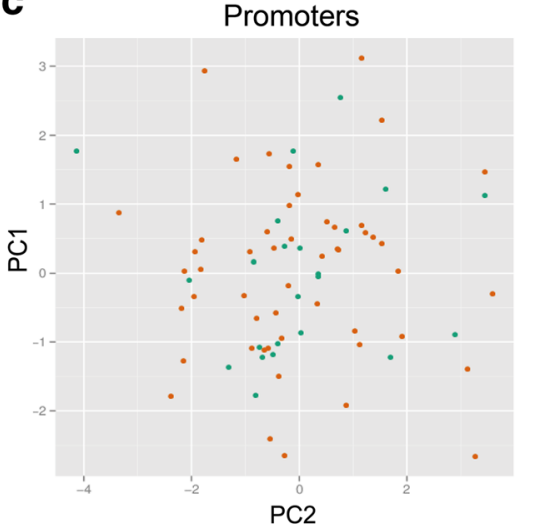

d

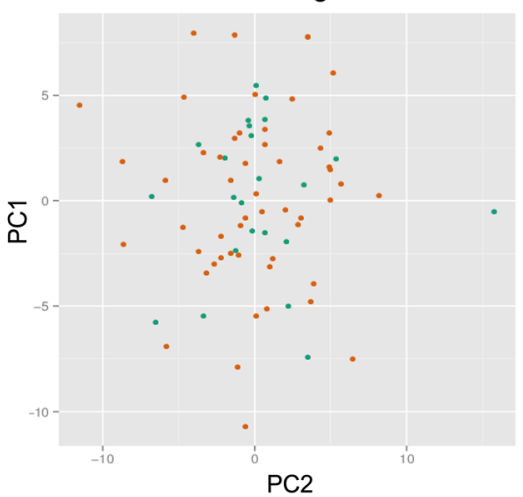

e

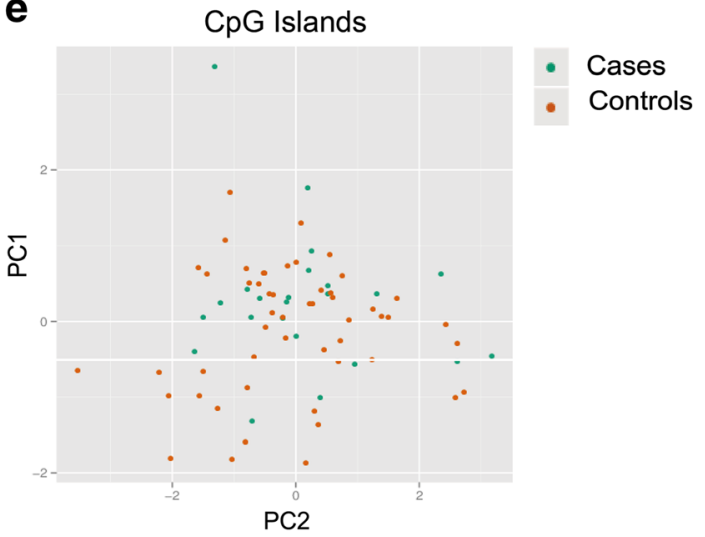

Fig. 2 Dimension reduction of methylation data. Dimension reduction is used to visually inspect the dataset for a strong signal in the methylation values that is related to sample clinical or chip batch effects. Values of the first two principal components (PC) in scatter plots are shown considering methylation levels of CpG sites (a) and methylation levels of regions such as genes (b), promoters (c), tiling (d), and CpG islands (e)

associated with the case-control status, and the chip batch were used as covariates to adjust the differential methylation analysis. Moreover, surrogate variables analysis (SVA) was applied in order to correct for potential unmeasured or unmodeled confounders. At site level, no statistical differences in methylation between cases and controls with a genome-wide approach $\left(p<10^{-7}\right)$ emerged. A list of the top 50 ranked probes as differently methylated is reported in Additional file 2: Table S1. Differential methylation analysis performed at regional level was conducted considering the $\mathrm{CpG}$ islands, promoters, genes, and tiling. After multiple testing correction, no statistical differences in region methylation levels emerged between cases and controls. QQ plots showing the level of inflation factor for adjusted and not adjusted analyses are reported in Additional file 3: Figure S2.

\section{Comparative analysis of differences in methylation status with previous EWAS}

The differential methylation analysis is based on the assumption that differences in methylation status are shared among subjects. We have thus evaluated the consensus among previous EWAS reporting differentially methylated genes in the cord blood between in vivo- and in vitro-conceived babies. The results presented with the Venn diagram in Additional file 4: Figure S3 showed a lack of consistency among the studies, with only 6 out of 214 genes found to be differentially methylated in at least two different studies (NAP1L5, L3MBTL, GNAS, PEG10, $P R C P$, and $R U N X 3)$. Importantly, none of these genes were differentially methylated between cases and controls in the present study, despite considering both adjusted and unadjusted $p$ values in the regional analysis conducted for genes and promoters. Considering all the 214 genes reported in the literature, only RNF185 [10] was found in our study to include one of the top 50 ranked probes reported in Additional file 2: Table S1. However, no enrichment of differentially methylated probes was observed for the gene RNF185 in our population, with only a single probe out of eight associated with the gene resulting to be nominally significant. Additionally, no differences were detected in the regional analysis for genes and promoters (unadjusted $p$ values: 0.09 and 0.19 for gene and promoter, respectively). 


\section{Stochastic epigenetic variations analysis}

For each subject, the total number of SEVs was calculated using three different reference populations as described in the "Methods" section. The three different estimations of the number of SEVs were then reported using a logarithmic scale and indicated as $\log (\mathrm{SEV}$ ). The median number of $\log (\mathrm{SEV})$ that was calculated using the naturally conceived cord blood reference was $5.9(\mathrm{Q} 1=5.8 ; \mathrm{Q} 2=6.6)$ in cases (in vitro-conceived babies) and 6.0 (Q1 =5.9; $\mathrm{Q} 2=$ 6.3) in controls (in vivo-conceived babies) (Fig. 3a). The median number of $\log (\mathrm{SEV})$ calculated using the naturally conceived cord blood GEO data reference was 7.5 $(\mathrm{Q} 1=7.3 ; \mathrm{Q} 2=7.7)$ in cases and 7.4 $(\mathrm{Q} 1=$ 7.2; $\mathrm{Q} 2=7.6$ ) in controls (Fig. 3b). The median number of $\log (\mathrm{SEVs})$ calculated using the general population whole blood reference was 7.4 $(\mathrm{Q} 1=7.2 ; \mathrm{Q} 2=$ 7.6) in cases and 7.4 $(\mathrm{Q} 1=7.2 ; \mathrm{Q} 2=7.6)$ in controls (Fig. 3c). For all the estimations, no statistically significant differences in $\log (\mathrm{SEVs})$ emerged between the two groups analyzed. Moreover, the distribution of $\log$ (SEVs) was very similar in cases and controls as shown in the densitograms in Fig. 3d-f. A multivariate logistic regression confirmed that no association was present between $\log (\mathrm{SEV} \mathrm{s})$ and case-control status after adjustment for the set of covariates used in the differential methylation analysis. Three different regression models were performed according to the three estimations of SEVs, and the results were finally combined using the Fisher's method. After multiple testing correction, Fisher's combined $P$ value was equal to 0.38 . An enrichment analysis was then conducted in order to identify, in each subject, the number of genomic regions that were enriched in SEVs. No differences between ICSI and naturally conceived babies were detected in the number of regions with enriched number of SEVs (Additional file 5: Figure S4A-C). Also the distribution of the number of regions enriched in SEVs number was similar between the two groups. (Additional file 5: Figure S4D-F).

\section{Stochastic epigenetic variations and imprinting defects}

We have also checked for the presence of regions enriched in SEVs in imprinted loci. The list of genes and genomic coordinates under imprinting was selected based on the paper by Court et al. [20]. The analysis identified the presence of a single subject with an enrichment of SEVs located at several differentially methylated regions (DMRs) in the locus GNAS (Additional file 6: Figure S5). In this subject from the control group, the high number of reported SEVs suggested a defect in the establishment or maintenance of methylation imprints. The epigenetic defect was confirmed using the methylation-specific multiplex ligation-dependent probe amplification (MS-MLPA) (data not shown).

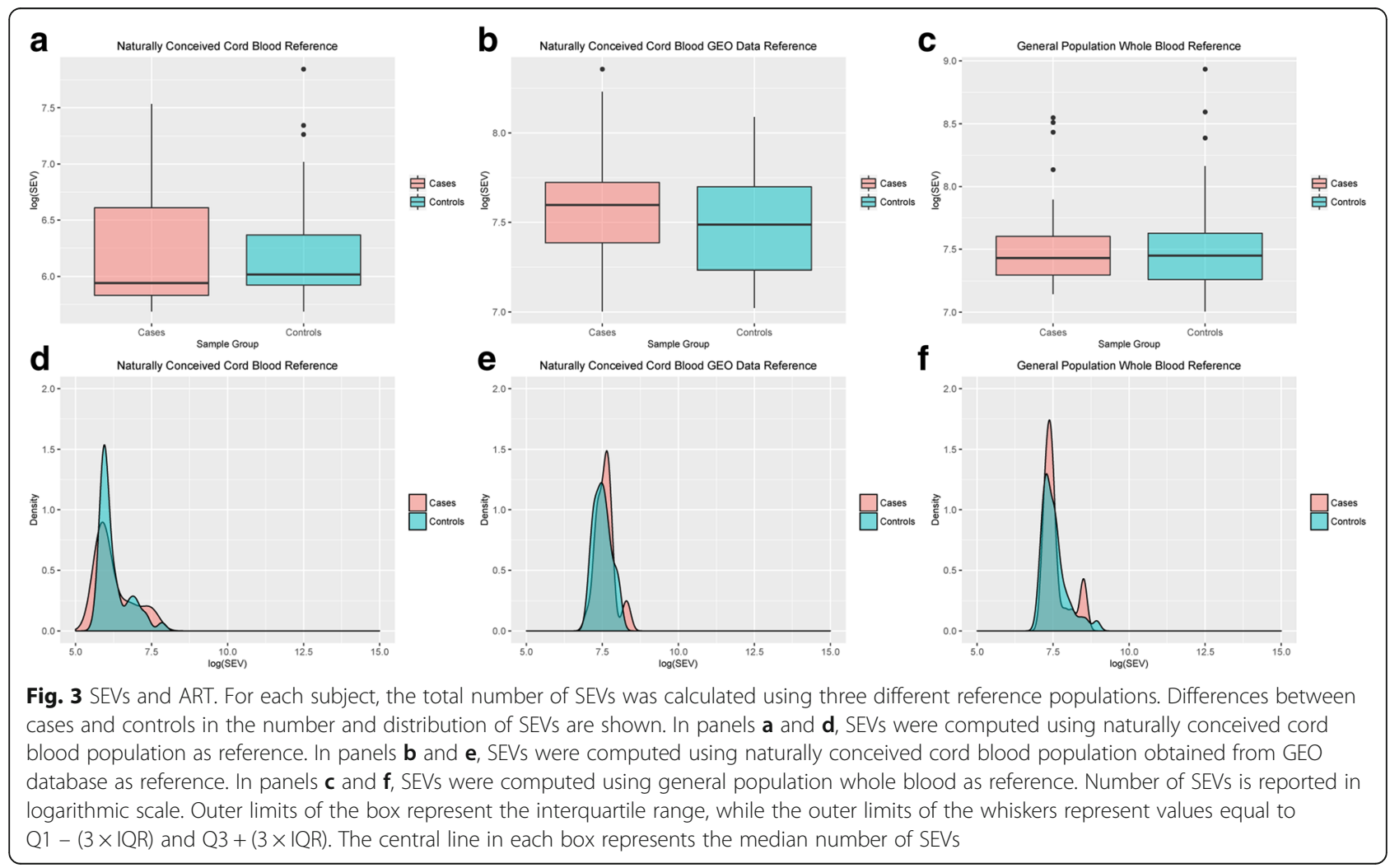


Stochastic epigenetic variations association analysis with phenotypic, behavioral, and environmental traits

A multivariate regression analysis was performed in order to identify phenotypic traits potentially associated with SEVs. Three different regression models were performed according to the three estimations of SEVs and the results were finally combined using Fisher's method. Each multivariate regression model was created considering the logarithmic number of SEVs as dependent variable and the first 10 dimensions obtained from the phenotypic multiple factor analysis as independent variables. Results were adjusted considering the chip batch effect. The multivariate regression showed that the Dim 1, Dim 4, Dim 5, and Dim 9 were strongly associated with the number of SEVs. After multiple testing correction, Fisher's combined $p$ values were $p=4.0 \times$ $10^{-4}, p=4.0 \times 10^{-4}, p=1.3 \times 10^{-3}$, and $p=5.0 \times 10^{-4}$, respectively. The phenotypic traits captured by those dimensions are shown in the correlogram in Fig. 1b. Birthweight centiles, gestational age, cesarean section, presence of adverse pregnancy outcomes, pre-gestational mother's weight, parents' BMI and obesity, and cord blood cells composition (CD8T, CD4T, and B Cells) were significantly correlated to $\operatorname{Dim} 1(p<0.01, \rho>\mid 0.5$ |). Birthweight centiles, mother's smoking status were significantly correlated to $\operatorname{Dim} 4(p<0.01, \rho>|0.5|)$, cord blood cells composition (NK cells) and season of birth were significantly correlated to Dim $5(p<0.01$, $\rho>|0.5|$ ) while Dim 9 captured mainly cesarean section and pre- or post-conception folates supplementation $(p<0.01, \rho>|0.4|)$. The four most significant traits observed to be correlated with SEVs (pre or post conception folates supplementation, cesarean section, mother's obesity status and season of birth) are shown in Additional file 7: Figure S6. The number of SEVs reported has been obtained using the naturally conceived cord blood reference.

\section{Discussion}

We report the most comprehensive multifactorial analysis of cord blood DNA methylation of in vitro conceived babies and the first analysis of the stochastic variations potentially induced by ART techniques. Although the use of ART has allowed millions of otherwise infertile couples to conceive children, some concern still remains about the safety of these procedures [2, 21]. An increase in imprinting disorders has been found in children conceived through IVF and ICSI, but no evidence of generalized changes in DNA methylation could be appreciated [2]. Our results are in line with this observation based on two different approaches: (i) a conventional epigenome-wide association analysis. At both site and region levels, no differences in methylation status between naturally and in vitro conceived emerged as statistically significant; (ii) a previously published method aimed at investigating the number and the localization of stochastic epigenetic variations [12, 22].

Although several studies have tried to investigate the methylation status of ART-conceived babies, only four EWAS were previously conducted on this topic. Melamed et al. have evaluated 27,578 CpG sites in cord blood from 10 children conceived in vitro and 8 conceived in vivo [10]. Castillo-Fernandez et al. investigated the links between IVF and DNA methylation patterns in 47 IVF and 60 non-IVF newborn twins (from 54 twin pairs) in whole cord blood cells and cord blood mononuclear cells using genome-wide methylated DNA immunoprecipitation coupled with deep sequencing [13]. Estill et al. reported the methylation profiles of neonatal blood spots of 137 babies conceived naturally or with four different ART techniques [14]. Finally, El Hajj N et al. evaluated the methylome of 48 babies conceived with ICSI [15]. Both Estill et al. and El Hajj $\mathrm{N}$ et al. used the Infinium HumanMethylation450 BeadChips to analyze the methylation levels $[14,15]$. Notwithstanding the lack of an adequate correction for crucial confounding factors, only a small number of CpG sites was differentially methylated at a genome-wide level and the magnitude of differences identified was also extremely small [10, 13-15]. Furthermore, when considering all the studies together, we observed a substantial inconsistence among their results. Only six genes have been confirmed in at least two studies (NAP1L5, L3MBTL, GNAS, PEG10, PRCP, and RUNX3).

Interestingly, a review of literature concerning those genes revealed that they are already known to be epigenetically associated with other pregnancy conditions. NAP1L5, for example, has been previously identified to be differently methylated in cord blood samples characterized by intrauterine exposure to gestational diabetes mellitus [23]. L3MBTL has been reported to be differently methylated in cord blood of babies exposed to smoking during pregnancy [24] and is epigenetically associated with gestational age [25]. Increased methylation at differentially methylated regions of GNAS has been described in infants born in conditions of gestational diabetes [26]. An increase in DNA methylation at the SGCE/PEG10 DMR has been previously positively associated with paternal BMI [27], maternal stress [28], and also with maternal prenatal physical activity [29]. Methylation of PRCP gene in newborns [30] as well as RUNX3 [24] was found to be significantly associated with maternal smoking status. Hypermethylation of RUNX3 CpG sites has been also associated with decreased gestational age [24]. Taken together, this evidence hints at the presence of confounding factors in previously reported results on epigenetics of ART. This observation seems to be supported by analysis of the 
QQ-plot obtained from unadjusted analysis presented herein. The elevated genomic inflation factor (lambda = 1.7; Additional file 3: Figure S2) observed in unadjusted analysis sheds light on the excess of false positive rate potentially produced under inadequate correction. The influence of confounding factors on epigenome of cord blood has been extensively described. Soubry et al., for example, demonstrated a significant association between parental BMI and DNA methylation of imprinted genes [29]. This finding should be considered as relevant since a significant effect of ART has been reported to affect imprinted loci [15].

An increased variability of DNA methylation in IVF conceived babies was previously underlined, hinting at the presence of epigenetic changes not shared among subjects [10] and thus supporting the idea that the effect of ART might be more likely to be stochastic rather than confined to some genetic regions. Our novel analytical approach addressing the number of stochastic epigenetic variations $[12,22]$ showed no differences in cord blood from in vitro and in vivo conceived babies.

The analysis of SEVs proposed in the present study proved to be a powerful approach to study the epigenetic variability and allowed to study the impact of several conditions on the newborn epigenome. Interestingly, four dimensions obtained from the dimensional reduction of phenotypic traits were significantly associated with the number of SEVs. Cord blood cell composition, pre or post conception supplementation of folates, birth percentiles, gestational age, cesarean section, pre-gestational mother's weight, parental BMI and obesity, presence of adverse pregnancy outcomes, mother's smoking status, and season of birth were the traits mainly captured by these dimensions. Some of these factors such as parental BMI, gestational age, birth weight, and smoking are already known for playing a role on the newborn epigenome [20, 25, 27$29,31]$. Of note, we observed and reported for the first time the existence of an epigenetic signature associated with season of birth. The number of stochastic epigenetic variations was lower in subjects born in autumn. This observation should be considered important for at least two reasons: (i) seasonality of birth has already been reported to be associated with an increased incidence of several pathological conditions such as type I diabetes [32, 33], cardiovascular disease [34], skin cancer [35], and autoimmune diseases [36]. Moreover, seasonality of birth has been also studied in the field of aging and is associated with life expectancy [37-40]; (ii) the magnitude of the effect of seasonality, a natural event, on epigenome of newborns appeared to be greater compared to the one induced by ART.

We have also evaluated the presence of SEVs in imprinted loci failing to find significant differences between cases and controls. A single epigenetic alteration in the locus GNAS was found in a control subject. It is important to note that the new method applied in this study allowed not only to evaluate the number of SEVs located across the whole genome, but also succeeded in the identification of a single epigenetic alteration subsequently confirmed using MS-MLPA and not shared among subjects. A standard case-control analysis would have failed to identify this particular defect. To our knowledge, this is the first study addressing a genome-wide analysis of imprinted loci taking into account the probable random effect of ART and applying a test that does not require the possible epigenetic variation to be present in more than one subject. In any case, we realize that these phenomena do represent rare events, and we probably need a greater sample size to completely exclude a direct effect at the level of specific imprinting genes.

The present work has clarified that the number of stochastic epigenetic variations potentially induced by ART technology was, at worst, comparable to that naturally produced in response to maternal behavior or other common environmental factors, thus debunking the idea of a severe impact of ART in the epigenome of the newborns and implying a reconsideration of the epigenetic safety of these techniques.

Another crucial aspect of the present work is the analysis of all the potential confounders that could affect the methylation status in the newborn cord blood. It is totally unexpected that previous studies on the same topic evaluating genome wide DNA methylation [10, 13-15] failed to correct for factors potentially affecting methylation status or considered only a small number of them. Therefore, given the complexity of the networks and of the phenotypic traits involved in pregnancy establishment, the causal relationship between the epigenetic status and ART needs to be evaluated with caution and controlling for potential confounders. The complexity of the phenotypic traits represents also another important issue; the correlation analysis performed among phenotypic traits revealed the existence of several hidden associations among variables, and this needs to be considered when adjusting for confounders in order to avoid multicollinearity.

Another aspect that is often disregarded is the potential role of ART techniques on placenta methylation status and the consequent effect on birth weight and on the epigenome of newborns. Recent studies conducted in animal models reported that ART has a predominant or exclusive effect on the placenta methylation status compared to that on the fetus [9, 41, 42]. An attractive hypothesis that comes from these data is that the higher incidence of premature birth and low birth weight observed in 
ART-conceived children may be related to abnormal placental function resulting from genomic imprinting errors at multiple genes. Recently, Ghosh et al. reported evidence of placental methylation altered by ART procedures at repeated sequences (LINE1 elements) and CCGG sites [43]. Similarly, Choux et al. reported lower DNA methylation for two imprinted loci (H19/IGF2 and KCNQ1OT1 DMRs) and two transposon families (LINE-1Hs and ERVFRD-1) in the placenta of babies conceived by ART [44]. Interestingly, DNA methylation of the same imprinted genes DMRs and transposable elements in cord blood was not altered by ART procedures, thus confirming the main effect at the placental level as seen in animal experiments. A whole genome analysis of the methylation status of placenta samples from babies conceived by ART is needed in order to elucidate this crucial aspect.

A number of limitations need to be considered when interpreting our results. First, only DNA from cord blood was analyzed. Since cord blood is not necessarily representative of the epigenetic status of all tissues and cells in the newborn, additional studies on other tissues are mandatory to confirm these data. Second, the phenotypic data of the studied subjects did not include all the possible variables related to pregnancy and labor although a surrogate variables analysis was conducted to compensate for this deficiency. Third, although the number of subjects used for comparisons is of the same order of magnitude of previously conducted EWAS, we must underline that a larger sample size could better estimate normal ranges of DNA methylation in each locus. Confirming our results on a larger sample size would allow to exclude the possibility that small methylation differences have gone undetected. Fourth, these findings represent the experience relative to the ICSI procedure of a single IVF center and a single lab. Although we selected only ICSI procedures in order to analyze a homogeneous group of babies obtained using the more invasive technique, we cannot exclude that procedures done in different ways and with different technologies could result in different data. Moreover, it should be considered that when comparing naturally conceived and in vitro-conceived babies, there is a consistent number of inescapable differences between the two groups such as parents' infertility status that may represent an important selection bias. Finally, although the Infinium HumanMethylation450 BeadChips covers 99\% of RefSeq genes, permitting a whole-genome analysis, the coverage of total CpG sites is low (around 2\%). This means that sequencing-based methods should be adopted in the future in order to study other genomic features (e.g., enhancers) that have only small coverage in the present analysis.

\section{Conclusions}

The presented prospective study does not support that children born after ART have an increased risk of genome-wide changes in DNA methylation neither at specific loci neither randomly scattered throughout the genome. On the other hand, the study confirms that there are several environmental and behavioral conditions able to affect epigenetic variability in cord blood and leads to the conclusion that they need to be considered as potential confounders in investigations of this nature.

A reanalysis of previous data based on phenotypic traits of the parents and the babies potentially associated with epigenetic changes is warranted as well as a meta-analysis including all the data from genome-wide studies.

\section{Methods}

\section{Study design and study population}

This is a prospective study designed to avoid biases related to the improper selection of complicated pregnancies in one of the two groups. Women that underwent ICSI treatment were enrolled in the study at 20 weeks' gestation. These women were stimulated with standard ovulation induction drugs. Pregnant women who naturally conceived were also enrolled at 20 weeks' of gestation. The in vivo group had no history of infertility, and the index pregnancy was achieved without medications or treatments. None of the pregnancy ended in abortion, and all the patients were included in the study. Samples of cord blood from both ART-conceived pregnancies $(n=23)$ and naturally conceived pregnancies $(n=41)$ were obtained at the time of delivery by the midwives of the San Raffaele Hospital, Milano, Italy, by puncturing the umbilical vein while the placenta was in utero [45]. Patients were informed that cord blood would be used for research purposes and gave written consent. Approval for this study was granted by the local Human Institutional Investigation Committee (\#PMAMET). Clinical information obtained for each pregnancy included demographic and obstetric factors, cause of infertility, details of the ART procedure as well as pregnancy, delivery, and neonatal outcomes. Multifetal gestations were excluded from both groups.

Sample size was calculated based on the analysis of parameters observed in a previous study in which more than 400 subjects were evaluated [12]. Mean and variance were estimated for all the CpG sites of the Infinium HumanMethylation 450 BeadChip array. The number of subjects to be enrolled has been calculated assuming an effect size taking into account a difference in the percentage of methylation between groups of at least $10 \%$, by imposing a probability of type I error in the order of $10^{-7}$ (level of significance that takes into account the 
need to correct for multiple testing) and a power of $95 \%$. A description of the study design is shown in Additional file 8: Figure S7.

\section{In vitro fertilization procedures}

Controlled ovarian stimulation was performed according to the clinical practice and as previously described [46-48]. Oocyte collection was performed $36 \mathrm{~h}$ after triggering of ovulation. After 2-3 h incubation in Human Serum Albumin (HSA)-supplemented Fertilization medium (Sage In-Vitro Fertilization, Inc. Trumbull, CT, USA) under oil, denudation of the cumulus oophorus was performed as previously described $[46,49,50]$. Injected oocytes were grouped-cultured in microdrops of equilibrated Serum Substitute Supplement (SSS, Irvine, CA, USA)-supplemented Cleavage medium (Sage In-Vitro Fertilization, Inc. Trumbull, CT, USA) under oil. Sixteen to $18 \mathrm{~h}$ after ICSI, all oocytes were checked for fertilization as previously described $[46,49,50]$. For a subgroup of patients $(n=7$, $30.4 \%$ ), embryos were cultured to blastocyst stage in SSS (Irvine, CA, USA)-supplemented Blastocyst medium (Sage In-Vitro Fertilization, Inc. Trumbull, CT, USA). All the incubation steps were conducted using low (5\%) oxygen concentration incubators [49]. All the transfers were performed in fresh cycles [48]. All the patients underwent luteal phase support with progesterone $600 \mathrm{mg} / \mathrm{d}$ (Prometrium) administered vaginally and continued through week 12 of pregnancy.

\section{DNA extraction and bisulphite treatment of the DNA}

Genomic DNA was extracted from cord blood using the Wizard genomic DNA purification kit (PROMEGA, Madison WI, USA) as previously described [12]. Quality control and quantification of DNA were performed before and after bisulphite conversion. DNA was quantified with NanoDrop (NanoDrop Products Thermo Scientific, Wilmington, DE, USA) and quality was assessed by visualization of genomic DNA on $1 \%$ agarose gel electrophoresis. Only DNA samples not fragmented and with a concentration higher than $50 \mathrm{ng} / \mu \mathrm{l}$ were subsequently processed.

\section{DNA methylation assay}

$4 \mu \mathrm{l}$ of bisulfite-converted DNA was used for hybridization on Infinium HumanMethylation 450 BeadChip, following the Illumina Infinium HD Methylation protocol. This consists of a whole genome amplification step followed by enzymatic end-point fragmentation, precipitation, and resuspension. The resuspended samples were hybridized on HumanMethylation 450 BeadChips at $48{ }^{\circ} \mathrm{C}$ for $16 \mathrm{~h}$. Then, unhybridized and non-specifically hybridized DNA were washed away followed by a single nucleotide extension using the hybridized bisulfite-treated DNA as a template. The nucleotides incorporated were labeled with biotin (ddCTP and ddGTP) and 2,4-dinitrophenol (DNP) (ddATP and ddTTP). After the single base extension, repeated rounds of staining were performed with a combination of antibodies that differentiate DNP and biotin by fixing them different fluorophores. Finally, the BeadChip was washed and protected in order to scan it. The Illumina HiScan SQ scanner is a two-color laser $(532 \mathrm{~nm} / 660 \mathrm{~nm})$ fluorescent scanner with a $0.375 \mu \mathrm{m}$ spatial resolution capable of exciting the fluorophores generated during the staining step of the protocol. Image intensities were extracted using GenomeStudio (2010.3). The methylation score for each $\mathrm{CpG}$ site was represented as $\beta$ values according to the fluorescent intensity ratio between methylated and unmethylated probes. $\beta$ values may range between 0 (unmethylated) and 1 (completely methylated).

\section{Data management, pre-processing, normalization, and quality control}

Illumina Methylation 450K raw data were analyzed using the RnBeads analysis software package [51]. Sites with overlapping SNPs were firstly removed from the analysis $(n=4713)$ as well as probes on sex chromosomes $(n=11119)$. Possible removal of probes and samples of highest impurity from the dataset was evaluated using the Greedycut algorithm. We considered every $\beta$ value to be unreliable when its corresponding detection $p$ value was not below the threshold $(T=0.05)$. In order to avoid an erroneous interpretation of stochastic epigenetic variations, probes with coordinates overlapping rare genetic variants annotated in 1000 genomes and EXAC databases were removed [52, 53]. After the quality control step, none of the samples was excluded for quality reasons while a total of 14208 probes were removed. The background was subtracted using the methylumi package (method "noob") [51]. The signal intensity values were normalized using the SWAN normalization method, as implemented in the minfi package. In addition to $\mathrm{CpG}$ sites, four sets of genomic regions were covered in the analysis (tiling, genes, promoters and $\mathrm{CpG}$ Islands).

\section{Blood cell type counts}

Proportions of CD8 T cells, CD4 T cells, NK cells, B cells, monocytes, and granulocytes were estimated using the "estimateCellCounts" function in the Bioconductor minfi package [54] with the reference data for cord blood provided by Bakulski et al. [55].

\section{Differential methylation analysis}

Differential methylation analysis was conducted both at site and region level according to the sample groups. $p$ values were computed using the limma method for the site level analysis while a combined $p$ value was 
calculated from all site $p$ values for the region-based [51]. Regions were defined according to RnBeads definitions [51]:

- Genes and promoters: Ensembl [56] gene definitions were downloaded using the biomaRt package. A promoter was defined as the region spanning 1500 bases upstream and 500 bases downstream of the transcription start site of the corresponding gene.

- GpG islands: the CpG island track was downloaded from the dedicated FTP directory of the UCSC Genome Browser [57].

- Tiling regions: not overlapping tiling regions with a window size of $5 \mathrm{~kb}$ were defined over the whole genome.

In order to avoid potentially confounding factors, a multiple factor analysis (MFA) was performed based on phenotypic data (birth weight, birthweight centiles, mother' s age, parity status, gestational age, cesarean section, sex of the baby, presence of adverse pregnancy outcomes, pre-gestational mother weight, parents' BMI and obesity status, gestational weight increase, smoking status, pre-post conception folate supplementation, CD8T, CD4T, NK cells, B cells, monocytes, granulocytes, season of birth). Variables were entered in the analysis as factorial or linear values. Chip batch and dimensions that were significantly associated with the case-control status were used as covariates in the differential methylation analysis. Adjustment for surrogate variables was conducted using the function directly provided in the package RnBeads, which can detect batch effects and other unwanted variation of unknown origin and annotate them in such a way that they can be controlled for as covariates [51].

\section{Epigenetic variations detection}

In order to identify stochastic epigenetic variations (SEVs), we used a method previously described by our group [12, 22]. Briefly, after the pre-processing step, the distribution and variability of methylation levels were studied in the populations for all the probes of the array using box and whiskers plots. At each CpG site, the methylation level of a subject that was extremely different from the rest of the population was counted as an epigenetic variation. Thus, for each locus, epigenetic variations were identified as the extreme outliers, with their methylation level that lied outside of $\mathrm{Q} 1-(3 \times \mathrm{IQR})$ and $\mathrm{Q} 3+(3 \times \mathrm{IQR})$. Finally, all the observed epigenetic variations were annotated in a new data matrix that allowed to calculate, for each subject, the total amount of epigenetic variations and their genomic position. The box and whiskers plot analysis was conducted using boxplot function in the R car package and confirmed using the outlier function in the R outliers package.
In addition to the previously described control population composed by 41 natural conceived babies, two other different reference populations have been used for this analysis:

1) Methylation row data from 60 subjects were obtained from the public functional genomics data repository GEO. We have selected two different datasets containing methylation data from cord blood samples (GSE54399, GSE30870), and we have extracted only methylation data from control subjects. This population (naturally conceived cord blood GEO data reference) represented our "external" control population. GEO and in house raw data were preprocessed following the same procedure.

2) Methylation row data from 350 healthy subjects with an age spanning from 1 to 107 years were also obtained from the Istituto Auxologico Italiano Epigenetic Database. This population represented a second reference population used for the estimation of epigenetic variations (general population whole blood reference).

A schematic description of the strategy used to obtain different estimations of the number of SEVs by using the various reference populations is shown is Additional file 9: Figure S8.

Briefly:

- In the first step, the analysis described above was applied on naturally conceived cord blood population. Samples were analyzed together, and the number of SEVs was calculated in each control subject.

- In a second step, all the samples in the case group were tested individually using the naturally conceived cord blood reference and the number of SEVs was calculated for each case subject.

- In the third step, the naturally conceived cord blood GEO data reference was used to calculate the number of SEVs both in cases and control subjects. Also in this step, each subject was tested individually.

- Finally, the general population whole blood reference was used to calculate the number of SEVs both in control and case subjects. Also in this step, each subject was tested individually.

Using three different reference populations, three different estimations of the number of SEVs were calculated for each subject. A test for over-representation of these probes was conducted, for each subject, using sliding windows and the hypergeometric cumulative function, obtaining the number of genomic regions that were enriched in SEVs [12]. 


\section{Validation of the SEVs analysis}

In order to confirm the power of this analytical approach to detect epigenetic variations, two separate tests were performed on positive controls. Three samples were analyzed in duplicates, and epigenetic variations found in each of them were compared. Results showed a mean correlation of $0.99(p<0.01)$ among the experiments. The duplicate samples underwent independent bisulfite conversion reactions, and this suggests that epigenetic variations are not significantly influenced by bisulfite conversion errors. In the second validation step, 48 whole blood DNA samples obtained from subjects affected by imprinting diseases (Beckwith-Wiedemann syndrome, Angelman syndrome and Silver Russel syndrome) who underwent diagnostic assays at Istituto Auxologico Italiano were analyzed. For these subjects, a medical report indicating the genomic position of their epigenetic alteration was already available. Briefly, after the identification of the outlier probes, a test for over-representation of these probes inside each gene was performed using the hypergeometric cumulative function. The analysis identified genes with enriched number of outliers probes (Bonferroni's corrected $p$ value $<0.05$ ) confirming the presence of the epigenetic alterations previously reported in the medical report.

\section{Statistical tests}

The "Shapiro.test" function provided in the $\mathrm{R}$ package "stats" was applied to test normality among variables. The "Wilcox.test" function provided in the R package "class" was used to test differences between cases and controls groups for all non-parametric data. Considering the presence of categorical variables, dimensional reduction was performed using the multiple factor analysis of mixed data approach and the "FAMD" function provided in the $\mathrm{R}$ package "FactoMineR." The univariate and multivariate linear regressions were conducted using the "Generalised Linear Model" function provided in the $\mathrm{R}$ "base" package. Bonferroni's correction was performed to correct for multiple testing. Correlation analysis between Dims and environmental and behavioral conditions has been performed using the hector function provided in the package "polycor."

\section{Comparative analysis with previous EWAS}

We conducted a systematic search of the literature focusing on studies that used a genome-wide approach (Illumina Infinium HumanMethylation27 BeadChip and Illumina Infinium Human Methylation 450K BeadChip) with the aim to compare cord blood methylation levels between in vitro and in vivo conceived babies. The four selected studies [10,13-15] reported differentially methylated genes using different parameters for the selection.
Melamed et al. identifies genes that had at least two significantly differentially methylated CpG sites and genes with at least a CpG site showing a significant methylation difference $\geq 10 \%$ between ART and control groups [10]. A total of 33 genes were found. Estill et al. considered as significant all those genes with a minimum absolute average methylation change of $2.5 \%$ for the clusters of CpGs that were associated with a given gene (at least $75 \%$ of the cluster intersecting a gene body or promoter), with only the significant clusters that were considered in the average count [14]. The study reported a list of differentially methylated metastable epialleles, imprinted genes, and genes related to differentially methylated enhancers. A total of 87 genes as having significantly different methylation status emerged when comparing babies born with various ART techniques and naturally conceived. Castillo-Fernandez et al. reported a list of all the genes located near each differentially methylated CpGs between the two groups selected using a false discovery rate equal to $25 \%$ [13]. A total of 66 genes were found to be associated with the 46 reported differentially methylated regions. Finally, El Hajj et al. reported a list of 34 genes with a methylation difference of $\beta> \pm 0.03$ and an adjusted $p<0.05$ observed in promoters, imprinting control regions and $\mathrm{CpG}$ islands [15].

\section{Additional files}

Additional file 1: Figure S1. Correlation analysis among phenotypic, behavioral and environmental features considered in the study. Degree and direction of correlations are highlighted by the color and dimension of squares. (TIF 307 kb)

Additional file 2: Table S1. Top 50 ranked hypomethylated or hypermethylated probes in babies born after ICSI. (XLS $41 \mathrm{~kb}$ )

Additional file 3: Figure S2. QQ plots obtained using (A) Dim1 and Dim3 resulted from the multiple factor analysis of mixed data. (B) Surrogate variable analysis (SVA), and Dim1 and Dim3 obtained from the multiple factor analysis of mixed data as covariates in the differential methylation analysis. The QQ plot obtained without data correction is illustrated in C. The elevated genomic inflation factor of unadjusted data suggests presence of potential confounders. (TIF $141 \mathrm{~kb}$ )

Additional file 4: Figure S3. Venn diagram illustrating the number of genes found in literature to be differentially methylated in cord blood of ART babies when compared to natural conceived babies and the overlapping of results among previous EWAS [10, 13-15]. (TIF $234 \mathrm{~kb}$ )

Additional file 5: Figure S4. For each subject, the total number of region enriched in SEVs was calculated using three different reference populations. Differences between cases and controls in the number and distribution of region enriched in SEVs are shown. In panels A and D, SEVs were computed using naturally conceived cord blood population as reference. In panels B and E, SEVs were computed using naturally conceived cord blood population obtained from GEO database as reference. In panels $\mathrm{C}$ and $\mathrm{F}$, SEVs were computed using general population whole blood as reference. Number of region enriched in SEVs is reported in logarithmic scale. Outer limits of the box represent the interquartile range, while the outer limits of the whiskers represent values equal to Q1 - $(3 \times 1 \mathrm{QR})$ and Q3 $+(3 \times$ IQR $)$. The central line in each box represents the median number of SEVs. (TIF $181 \mathrm{~kb}$ ) 
Additional file 6: Figure S5. Genomic regions under imprinting control carrying an epigenetic alteration in a control subject. In panel A, stochastic epigenetic variations (SEVs) detected in the cord blood of a single subject from the control population are represented in red while the differentially methylated regions (DMRs) reported by Court et al. [18] are represented in blue. The high number of reported SEVs suggested a defect in the establishment or maintenance of methylation imprints confirmed using MS-MLPA. Panel B illustrates the magnification of one of the DMRs. (TIF $1062 \mathrm{~kb}$ )

Additional file 7: Figure S6. Effect of folates supplementation, mother's obesity status, cesarean section, and season of birth on number of SEVs. Number of SEVs is reported in logarithmic scale. Outer limits of the box represent the interquartile range, while the outer limits of the whiskers represent values equal to $\mathrm{Q} 1-(3 \times \mathrm{IQR})$ and $\mathrm{Q} 3+(3 \times \mid \mathrm{QR})$. The central line in each box represents the median number of SEVs. (TIF $107 \mathrm{~kb}$ )

Additional file 8: Figure S7. Schematic representation of the study design. (TIF $1116 \mathrm{~kb}$ )

Additional file 9: Figure S8. Schematic description of the strategy used to estimate the number of SEVs. (TIF $982 \mathrm{~kb}$ )

\section{Abbreviation}

ART: Assisted reproduction technology; BMl: Body mass index; Dim: Dimension; DMR: Differentially methylated region; EWAS: Epigenome wide association study; ICSI: Intra citoplasmatic sperm injection; IVF: in vitro fertilization; NGS: Next generation sequencing; SEV: Stochastic epigenetic variation; SVA: Surrogate variable analysis

\section{Availability of data and materials}

The datasets used and analyzed during the current study are available from the corresponding author on reasonable request.

\section{Authors' contributions}

$D G, E S, P G, L B, E P, M C, A M D B$, and PV planned and implemented the study LP and ER collected the data. DG does the statistical analyses. DG and PV wrote the first draft of the manuscript. All authors participated to the discussion of the findings and revised the manuscript. All authors read and approved the final manuscript.

\section{Ethics approval and consent to participate}

The study was approved by the local Human Institutional Investigation Committee (\#PMAMET).

\section{Competing interests}

Dr. Davide Gentilini, Dr. Luca Pagliardini, Dr. Elisa Rabellotti, Dr. Paolo Garagnani, Prof. Luisa Bernardinelli, Dr. Anna Maria Di Blasio, and Dr. Paola Vigano' declare no COls in relation to the work described. Prof. Edgardo Somigliana declares no personal COls. His institution received grants for research from Ferring, Merck Serono, and IBSA. Dr. Enrico Papaleo reports consultancies with MSD, Merck-Serono, Ferring, and IBSA Institut Biochimique SA; grants for institutional research from MSD, Merck-Serono, Ferring, and IBSA Institut Biochimique SA; honoraria from MSD, Merck-Serono; and travel expenses paid by MSD, Merck-Serono, Ferring, and IBSA Institut Biochimique SA. Prof. Candiani Massimo reports grants for institutional research from Merck-Serono, Astella Pharma, Roche Diagnostics, MSD Italia, Ferring.

\section{Publisher's Note}

Springer Nature remains neutral with regard to jurisdictional claims in published maps and institutional affiliations.

\section{Author details}

${ }^{1}$ Istituto Auxologico Italiano IRCCS, 20095 Cusano Milanino, Italy. ${ }^{2}$ Infertility Unit, Fondazione Ca' Granda, Ospedale Maggiore Policlinico, 20122 Milan, Italy. ${ }^{3}$ Reproductive Sciences Laboratory, Division of Genetics and Cell Biology, IRCCS Ospedale San Raffaele, Via Olgettina 58, 20132 Milan, Italy. ${ }^{4}$ Department of Experimental, Diagnostic and Specialty Medicine, University of Bologna, 40138 Bologna, Italy. ${ }^{5}$ Department of Brain and Behavioral Sciences, University of Pavia, 27100 Pavia, Italy. ${ }^{6}$ Obstetrics and Gynaecology Unit, IRCCS Ospedale San Raffaele, 20132 Milan, Italy.
Received: 27 November 2017 Accepted: 29 May 2018

Published online: 08 June 2018

\section{References}

1. $\quad$ liadou AN, Janson PC, Cnattingius S. Epigenetics and assisted reproductive technology. J Intern Med. 2011;270:414-20.

2. Lazaraviciute G, Kauser M, Bhattacharya S, Haggarty P, Bhattacharya S. A systematic review and meta-analysis of DNA methylation levels and imprinting disorders in children conceived by IVF/ICSI compared with children conceived spontaneously. Hum Reprod Update. 2014;20:840-52.

3. Song S, Ghosh J, Mainigi M, Turan N, Weinerman R, Truongcao M, et al DNA methylation differences between in vitro- and in vivo-conceived children are associated with ART procedures rather than infertility. Clin Epigenetics. 2015;7:41-9.

4. Odom LN, Segars J. Imprinting disorders and assisted reproductive technology. Curr Opin Endocrinol Diabetes Obes. 2010;17:517-22.

5. Tierling S, Souren NY, Gries J, Loporto C, Groth M, Lutsik P, et al. Assisted reproductive technologies do not enhance the variability of DNA methylation imprints in human. J Med Genet. 2010;47:371-6.

6. De Waal E, Yamazaki Y, Ingale P, Bartolomei M, Yanagimachi R, McCarrey JR. Primary epimutations introduced during intracytoplasmic sperm injection (ICSI) are corrected by germline-specific epigenetic reprogramming. Proc Natl Acad Sci U S A. 2012;109:4163-8.

7. Batcheller A, Cardozo E, Maguire M, DeCherney AH, Segars JH. Are there subtle genome-wide epigenetic alterations in normal offspring conceived by assisted reproductive technologies? Fertil Steril. 2011;96: 1306-11

8. Messerschmidt DM, Knowles BB, Solter D. DNA methylation dynamics during epigenetic reprogramming in the germline and preimplantation embryos. Genes Dev. 2014;28:812-28.

9. De Waal E, Mak W, Calhoun S, Stein P, Ord T, Krapp C, et al. In vitro culture increases the frequency of stochastic epigenetic errors at imprinted genes in placental tissues from mouse concepti produced through assisted reproductive technologies. Biol Reprod. 2014;90:22.

10. Melamed N, Choufani S, Wilkins-Haug LE, Koren G, Weksberg R. Comparison of genome-wide and gene-specific DNA methylation between ART and naturally conceived pregnancies. Epigenetics. 2015;10:474-83.

11. Arnaud P, Hata K, Kaneda M, Li E, Sasaki H, Feil R, et al. Stochastic imprinting in the progeny of Dnmt3L-/- females. Hum Mol Genet. 2006;15:589-98.

12. Gentilini D, Garagnani P, Pisoni S, Bacalini MG, Calzari L, Mari D, et al. Stochastic epigenetic mutations (DNA methylation) increase exponentially in human aging and correlate with X chromosome inactivation skewing in females. Aging. 2015;7:568-78.

13. Castillo-Fernandez JE, Loke YJ, Bass-Stringer S, Gao F, Xia Y, Wu H, et al. DNA methylation changes at infertility genes in newborn twins conceived by in vitro fertilisation. Genome Med. 2017:9.28.

14. Estill MS, Bolnick JM, Waterland RA, Bolnick AD, Diamond MP, Krawetz SA Assisted reproductive technology alters deoxyribonucleic acid methylation profiles in bloodspots of newborn infants. Fertil Steril. 2016;106:629-39.

15. El Haij N, Haertle L, Dittrich M, Denk S, Lehnen H, Hahn T, et al. DNA methylation signatures in cord blood of ICSI children. Hum Reprod. 2017;32: $1761-9$

16. Diplas Al, Lambertini L, Lee MJ, Sperling R, Lee $Y L$, Wetmur J, et al. Differential expression of imprinted genes in normal and IUGR human placentas. Epigenetics. 2009:4:235-40.

17. Turan N, Ghalwash MF, Katari S, Coutifaris C, Obradovic Z, Sapienza C. DNA methylation differences at growth related genes correlate with birth weight: a molecular signature linked to developmental origins of adult disease? BMC Med Genet. 2012;5:10

18. Joubert BR, Felix JF, Yousefi P, Bakulski KM, Just AC, Breton C, et al. DNA methylation in newborns and maternal smoking in pregnancy: genomewide consortium meta-analysis. Am J Hum Genet. 2016;98:680-96.

19. Sharp GC, Salas LA, Monnereau C, Allard C, Yousefi P, Everson TM, et al. Maternal BMI at the start of pregnancy and offspring epigenome-wide DNA methylation: findings from the pregnancy and childhood epigenetics (PACE) consortium. Hum Mol Genet. 2017;26:4067-85.

20. Court F, Tayama C, Romanelli V, Martin-Trujillo A, Iglesias-Platas I, Okamura $\mathrm{K}$, et al. Genome-wide parent-of-origin DNA methylation analysis reveals the intricacies of human imprinting and suggests a germline methylationindependent mechanism of establishment. Genome Res. 2014;24:554-69. 
21. Rubino $P$, Viganò $P$, Luddi $A$, Piomboni $P$. The ICSI procedure from past to future: a systematic review of the more controversial aspects. Hum Reprod Update. 2016;22:194-227.

22. Gentilini D, Scala S, Gaudenzi G, Garagnani P, Capri M, Cescon M, et al. Epigenome-wide association study in hepatocellular carcinoma: identification of stochastic epigenetic mutations through an innovative statistical approach. Oncotarget. 2017:8:41890-902.

23. Haertle L, El Hajj N, Dittrich M, Müller T, Nanda I, Lehnen H, et al. Epigenetic signatures of gestational diabetes mellitus on cord blood methylation. Clin Epigenetics. 2017;9:28.

24. Maccani JZ, Koestler DC, Houseman EA, Marsit CJ, Kelsey KT. Placental DNA methylation alterations associated with maternal tobacco smoking at the RUNX3 gene are also associated with gestational age. Epigenomics. 2013;5(6):619-30.

25. Yuen RK, Jiang R, Peñaherrera MS, McFadden DE, Robinson WP. Genome-wide mapping of imprinted differentially methylated regions by DNA methylation profiling of human placentas from triploidies. Epigenetics Chromatin. 2011:4:10.

26. Chen D, Zhang A, Fang M, Fang R, Ge J, Jiang $Y$, et al. Increased methylation at differentially methylated region of GNAS in infants born to gestational diabetes. BMC Med Genet. 2014;15:108.

27. Soubry A, Murphy SK, Wang F, Huang Z, Vidal AC, Fuemmeler BF, et al. Newborns of obese parents have altered DNA methylation patterns at imprinted genes. Int J Obes. 2015;39:650-7.

28. Vidal AC, Benjamin Neelon SE, Liu Y, Tuli AM, Fuemmeler BF, Hoyo C, Murtha AP, et al. Maternal stress, preterm birth, and DNA methylation at imprint regulatory sequences in humans. Genet Epigenet. 2014;6: 37-44.

29. McCullough LE, Mendez MA, Miller EE, Murtha AP, Murphy SK, Hoyo C. Associations between prenatal physical activity, birth weight, and DNA methylation at genomically imprinted domains in a multiethnic newborn cohort. Epigenetics. 2015;10:597-606.

30. Rotroff DM, Joubert BR, Marvel SW, Håberg SE, Wu MC, Nilsen RM, et al. A maternal smoking impacts key biological pathways in newborns through epigenetic modification in utero. BMC Genomics. 2016:17(1):976.

31. Chango A, Pogribny IP. Considering maternal dietary modulators for epigenetic regulation and programming of the fetal epigenome. Nutrients. 2015;7:2748-70

32. Kahn HS, Morgan TM, Case LD, Dabelea D, Mayer-Davis EJ, Lawrence JM et al. Association of type 1 diabetes with month of birth among U.S. youth: the SEARCH for diabetes in youth study. Diabetes Care. 2009;32:2010-5.

33. Vaiserman AM, Carstensen B, Voitenko VP, Tronko MD, Kravchenko VI, Khalangot MD, et al. Seasonality of birth in children and young adults (0-29 years) with type 1 diabetes in Ukraine. Diabetologia. 2007;50:32-5.

34. Li L, Boland MR, Miotto R, Tatonetti NP, Dudley JT. Replicating cardiovascular condition-birth month associations. Sci Rep. 2016:6:33166

35. La Rosa F, Liso A, Bianconi F, Duca E, Stracci F. Seasonal variation in the month of birth in patients with skin cancer. Br J Cancer. 2014;111:1810-3.

36. Pazderska A, Fichna M, Mitchel AL, Napier CM, Gan E, Ruchała M, et al. Impact of month of birth on the risk of development of autoimmune Addison's disease. J Clin Endocrinol Metab. 2016;101:4214-8.

37. Doblhammer G, Vaupel JW. Lifespan depends on month of birth. Proc Natl Acad Sci U S A. 2001;98(5):2934-9.

38. Ueda P, Edstedt Bonamy AK, Granath F, Cnattingius S. Month of birth and mortality in Sweden: a nation-wide population-based cohort study. PLoS One. 2013;8:e56425.

39. Ueda P, Edstedt Bonamy AK, Granath F, Cnattingius S. Month of birth and cause-specific mortality between 50 and 80 years: a population-based longitudinal cohort study in Sweden. Eur J Epidemiol. 2014;29:89-94.

40. Gavrilov LA, Gavrilova NS. Season of birth and exceptional longevity: comparative study of american centenarians, their siblings, and spouses. J Aging Res. 2011;2011:104616

41. De Waal E, Vrooman LA, Fischer E, Ord T, Mainigi MA, Coutifaris C, et al. The cumulative effect of assisted reproduction procedures on placental development and epigenetic perturbations in a mouse model. Hum Mol Genet. 2015;24:6975-85.

42. Li B, Chen S, Tang N, Xiao X, Huang J, Jiang F, et al. Assisted reproduction causes reduced fetal growth associated with downregulation of paternally expressed imprinted genes that enhance fetal growth in mice. Biol Reprod. 2016;94:45.
43. Ghosh J, Coutifaris C, Sapienza C, Mainigi M. Global DNA methylation levels are altered by modifiable clinical manipulations in assisted reproductive technologies. Clin Epigenetics. 2017;6:9-14.

44. Choux C, Binquet C, Carmignac V, Bruno C, Chapusot C, Barberet J, et al. The epigenetic control of transposable elements and imprinted genes in newborns is affected by the mode of conception: ART versus spontaneous conception without underlying infertility. Hum Reprod. 2018;33:331-40.

45. Solves P, Moraga R, Saucedo E, Perales A, Soler MA, Larrea L, et al. Comparison between two strategies for umbilical cord blood collection. Bone Marrow Transplant. 2003;31:269-73.

46. Restelli L, Paffoni A, Corti L, Rabellotti E, Mangiarini A, Vigano P, et al. The strategy of group embryo culture based on pronuclear pattern on blastocyst development: a two center analysis. J Assist Reprod Genet. 2014; 31:1629-34

47. Intra G, Alteri A, Corti L, Rabellotti E, Papaleo E, Restelli L, et al. Application of failure mode and effect analysis in an assisted reproduction technology laboratory. Reprod BioMed Online. 2016;33:132-9.

48. Papaleo E, Pagliardini L, Vanni VS, Delprato D, Rubino P, Candiani M, et al. A direct healthcare cost analysis of the cryopreserved versus fresh transfer policy at the blastocyst stage. Reprod BioMed Online. 2017;34:19-26.

49. Calzi F, Papaleo E, Rabellotti E, Ottolina J, Vailati S, Vigano P, et al. Exposure of embryos to oxygen at low concentration in a cleavage stage transfer program: reproductive outcomes in a time-series analysis. Clin Lab. 2012;58: 997-1003.

50. Corti L, Papaleo E, Pagliardini L, Rabellotti E, Molgora M, La Marca A, et al. Fresh blastocyst transfer as a clinical approach to overcome the detrimental effect of progesterone elevation at hCG triggering: a strategy in the context of the Italian law. Eur J Obstet Gynecol Reprod Biol. 2013;171:73-7.

51. Assenov Y, Muller F, Lutsik P, Walter J, Lengauer T, Bock C. Comprehensive analysis of DNA methylation data with RnBeads. Nat Methods. 2014;11: 1138-40.

52. 1000 Genomes Project Consortium, Auton A, Brooks LD, Durbin RM, Garrison EP, Kang HM, et al. A global reference for human genetic variation. Nature. 2015;526:68-74.

53. Lek M, Karczewski KJ, Minikel EV, Samocha KE, Banks E, Fennell T, et al. Analysis of protein-coding genetic variation in 60,706 humans. Nature. 2016; 536:285-91.

54. Aryee MJ, Jaffe AE, Corrada-Bravo H, Ladd-Acosta C, Feinberg AP, Hansen $K D$, et al. Minfi: a flexible and comprehensive Bioconductor package for the analysis of Infinium DNA methylation microarrays. Bioinformatics. 2014;30: 1363-9.

55. Bakulski KM, Feinberg Jl, Andrews SV, Yang J, Brown S, McKenney SL, et al. DNA methylation of cord blood cell types: applications for mixed cell birth studies. Epigenetics. 2016;11:354-62.

56. Ensembl. http://www.ensembl.org/index.html. Accessed 27 Nov 2017.

57. UCSC Genome Browser. http://genome.ucsc.edu/. Accessed 27 Nov 2017.

\section{Ready to submit your research? Choose BMC and benefit from:}

- fast, convenient online submission

- thorough peer review by experienced researchers in your field

- rapid publication on acceptance

- support for research data, including large and complex data types

- gold Open Access which fosters wider collaboration and increased citations

- maximum visibility for your research: over $100 \mathrm{M}$ website views per year

At BMC, research is always in progress.

Learn more biomedcentral.com/submissions 